Document downloaded from:

http://hdl.handle.net/10251/162566

This paper must be cited as:

Seimetz, M.; Bellido, P.; Mur, P.; Lera, R.; Ruiz-De La Cruz, A.; Sánchez, I.; Zaffino, R.... (2020). Electromagnetic pulse generation in laser-proton acceleration from conductive and dielectric targets. Plasma Physics and Controlled Fusion. 62(11):1-9.

https://doi.org/10.1088/1361-6587/abb2e5

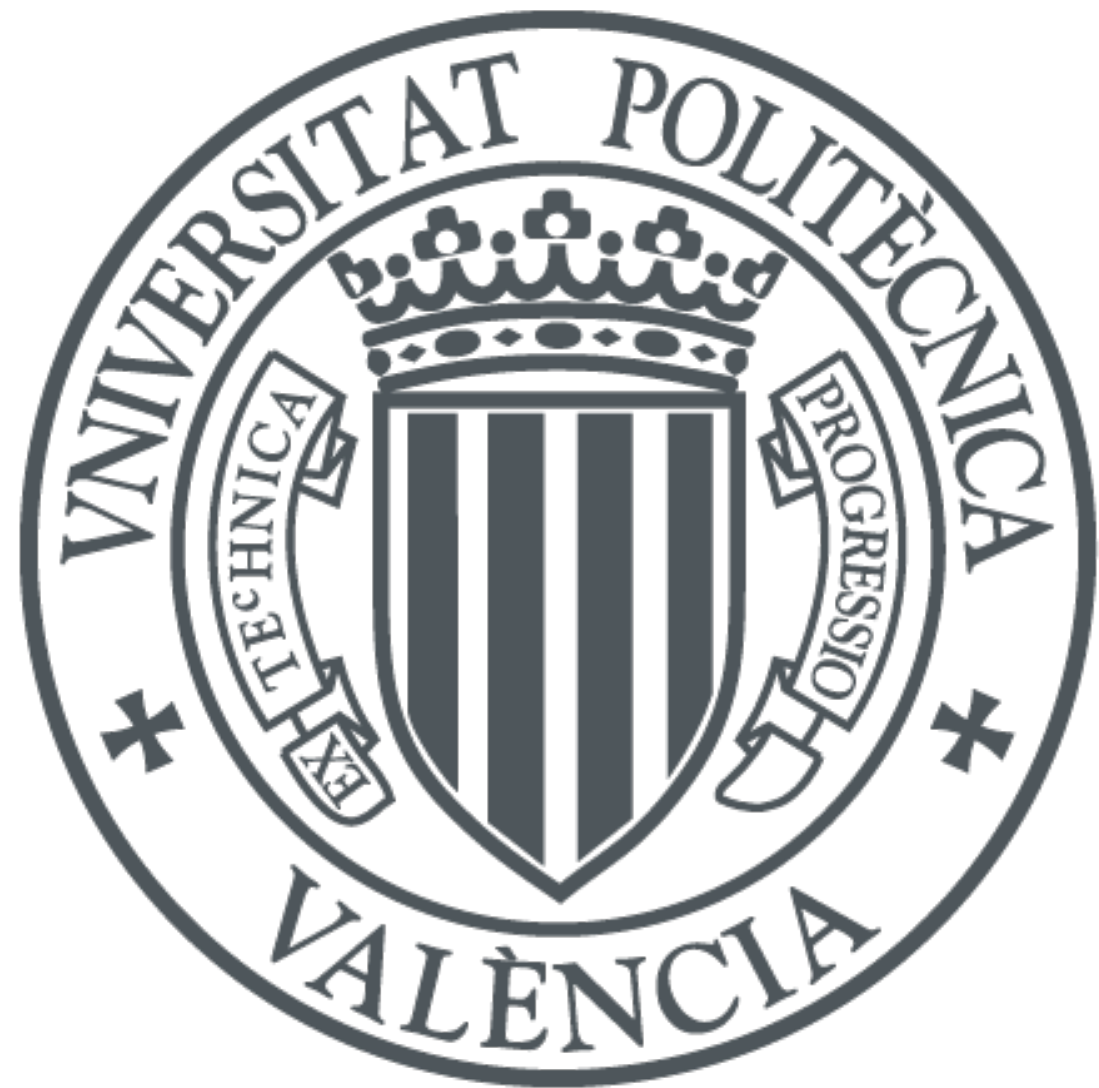

The final publication is available at

https://doi.org/10.1088/1361-6587/abb2e5

Copyright IOP Publishing

Additional Information 


\title{
Electromagnetic pulse generation in laser-proton acceleration from conductive and dielectric targets
}

\author{
M Seimetz ${ }^{1}, \mathbf{P}$ Bellido ${ }^{1,2,6}, \mathbf{P}$ Mur $^{1}, \mathbf{R}$ Lera $^{2}$, \\ A Ruiz-de la $\mathrm{Cruz}^{2}$, I Sánchez ${ }^{2}, \mathbf{R} \mathrm{Zaffino}^{3}$, J Benlliure ${ }^{4}$, \\ C Ruiz ${ }^{5}$, L Roso ${ }^{6}$ and JM Benlloch ${ }^{1}$ \\ ${ }^{1}$ Instituto de Instrumentación para Imagen Molecular (I3M), CSIC - Universitat \\ Politècnica de València, Camino de Vera s/n, Ed. 8B-N-1, 46022 Valencia, Spain \\ ${ }^{2}$ Proton Laser Applications S.L., Avda. Vilafranca del Penedès 11, 08734 \\ Olèrdola, Spain \\ ${ }^{3}$ Instituto de Microelectrónica de Barcelona (IMB-CNM, CSIC), C/ dels Til.lers \\ Campus UAB, 08193 Cerdanyola del Vallès (Barcelona), Spain \\ ${ }^{4}$ Instituto Galego de Física de Altas Enerxías (IGFAE), Universidade de \\ Santiago de Compostela, Rúa de Xoaquín Díaz de Rábago, 15705 Santiago de \\ Compostela, Spain \\ ${ }^{5}$ Instituto de Física Fundamental y Matemáticas, Universidad de Salamanca, \\ Casas del Parque, 37008 Salamanca, Spain \\ ${ }^{6}$ Centro de Láseres Pulsados (CLPU), Calle del Adaja, 37185 Villamayor, Spain \\ E-mail: mseimetz@i3m.upv.es
}

\begin{abstract}
Laser-plasma interactions at high intensities are often accompanied by emission of a strong electromagnetic pulse (EMP) interfering with particle detectors or other electronic equipment. We present experimental evidence for significant differences in noise amplitudes in laser-proton acceleration from aluminium as compared to mylar target foils. Such dissimilarities have been consistently observed throughout two series of measurements indicating that, under otherwise identical conditions, the target conductivity is the principal parameter related to EMP generation. In addition, the lateral size of the target foils correlates with the absolute noise levels. A frequency analysis combined with numerical simulations allows for an identification of several sources of radiofrequency emission in the $\mathrm{MHz}-\mathrm{GHz}$ regime. Further, the temporal evolution of single frequencies on the nanosecond scale provides information on distinct excitation mechanisms.
\end{abstract}

Keywords: Laser-ion acceleration, laser-plasma interaction, electromagnetic pulse, EMP, radiofrequency 


\section{Introduction}

Ion acceleration by focalisation of highly intense laser pulses on thin targets has become a very active field of experimental and theoretical research $[1,2]$. Among different interaction mechanisms described in the literature, Target Normal Sheath Acceleration (TNSA) $[3,4]$ has been most widely explored. Laser driven ion acceleration may give rise to applications, e.g., in particle therapy [5]. However, several important topics still need to be carefully addressed such as the particle energy and spectral distribution, the combination of high laser power and shot rate, and advanced target design and replacement. In this context, the generation of a strong electromagnetic pulse (EMP) has often been reported as a side effect in interactions of high-power, pulsed lasers with solid targets. Its amplitudes can be sufficiently large to disturb or harm electronic devices inside or outside the vacuum system [6]. The understanding and control of EMP has attracted considerable attention, both theoretical and experimental, not only due to its practical importance but also as a probe for laserplasma interaction processes. Precise, time-resolved analyses of the pulse structure have been obtained by dedicated measurements [7-11] and electronic solutions for EMP suppression have been proposed [12-15]. On the other hand, a possible application of the strong electromagnetic field for a two-step ion acceleration process has been discussed [16].

The EMP originates from various, independent sources, including electromagnetic oscillations of the vacuum chambers due to the impact of high-energy electrons or ions from the evaporating target $[17,18]$, return currents in the plasma plume around the laser interaction region $[19,20]$, and neutralisation currents inside the laser target and target holder shortly after the pulse [9,21-23]. These correspond to distinct frequency regimes in the $\mathrm{MHz}-\mathrm{THz}$ range. Some aspects of EMP generation have been addressed in dedicated models [21, 23-26].

Most of the experimental results have been obtained from (thick or thin) metallic targets. Strong EMPs have been observed from polymer targets as well [27]. Direct comparison between conductive and dielectric target materials showed quantitative differences in radiofrequency (rf) emission but not completely distinct patterns $[22,28-30]$. From a solid hydrogen ribbon, to the contrary, a strong suppression of EMP with respect to copper has been reported [31]. Evidence for especially low EMP generation from lowdensity gas targets has been found [32]. A correlation with the target geometry has also been inferred [33].

Here we report on experimental evidence for significantly lower EMP noise levels from dielectric (mylar) target foils as compared to conductive ones (aluminium). This has been consistently observed in two systematic series of laser-proton acceleration experiments (section 3). Different sources of $\mathrm{rf}$ emission are separated by Fourier analysis of the recorded signals and a comparison to numerical calculations of resonant eigenmodes of our setup is presented (section 4). Finally, we analye the temporal evolution of selected frequencies which allows for discrimination between two regimes of excitation (section 5).

\section{Experiment setup}

Our measurements have been performed using a 3 TW, table-top Ti:Sapphire laser developed by Proton Laser Applications, S.L. [34]. The laser energy was of 265$280 \mathrm{~mJ}$ (165-175 mJ on target) in pulses of $55 \mathrm{fs}$ duration (FWHM). It was focalized on thin target foils by a $60^{\circ}, \mathrm{f} / 3$ off-axis parabolic mirror with a spot size of $5.0 \times 11.5 \mathrm{\mu m}^{2}$, corresponding to peak intensities of the order $4 \times 10^{18} \mathrm{~W} / \mathrm{cm}^{2}$. The level of spurious prepulses was around $1: 10 \times 10^{-6}$ with respect to the peak intensity and the contrast over ASE was of the order $10^{-8}$ [35]. The repetition rate was of $0.5 \mathrm{~Hz}$ to the most, far below the intrinsic rate capability of the laser system $(10-100 \mathrm{~Hz})$.

The experiments reported here have been realized with flat foil targets. Target materials comprised

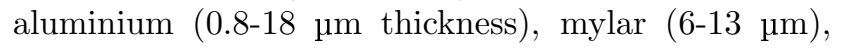
and aluminized mylar $(2 \mu \mathrm{m}$ bulk thickness with $50 \mathrm{~nm}$ of aluminium on the laser incidence side). Two distinct target holders have been used (Fig. 1), one with individual, circular sectors for operation in singleshot mode, and the other with an elongate area for repeated shots at $0.25-0.5 \mathrm{~Hz}$ repetition rate during continuous target movement. The focal position was adjusted by imaging the retroreflected speckle pattern of an auxiliary laser either for each shot or, in case of multiple shots, once before each series. The laser energy on target was slightly higher with multi-shot foils $(175 \mathrm{~mJ})$ than with single-shot targets $(165 \mathrm{~mJ})$.

(a)

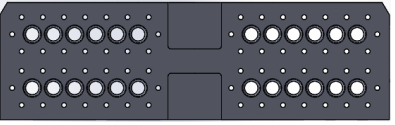

(b)

Figure 1. (a) Target holder for 24 individual, flat foil targets with $5 \mathrm{~mm}$ diameter. (b) Holder for two elongate foils of $6 \mathrm{~cm}$ length.

Accelerated protons have been detected with the time-of-flight (TOF) method by use of a fast plastic scintillator coupled to a photomultiplier tube (PMT, type Hamamatsu R647) via optical fibers [36]. Variable neutral density filters (OD1-OD2) between the fiber 
end and the PMT cathode window allow for adjusting the dynamic range. The scintillator was located in target normal direction $227 \mathrm{~cm}$ behind the laser target. All parts of the TOF detector were mounted inside a vacuum system (Fig. 2) connected to the interaction chamber, with the PMT protected from direct target view. A dipole magnet $(0.04 \mathrm{~T}) 10 \mathrm{~cm}$ in front of the scintillator swept away electrons with energies below $1.5 \mathrm{MeV}$ while protons of $E_{p}>$ $0.1 \mathrm{MeV}$ hit the detector. The PMT anode signals were recorded on a fast oscilloscope (Tektronix DPO 4102B, $1 \mathrm{GHz}, 5 \mathrm{GS} / \mathrm{s}$ ) which was located about $1 \mathrm{~m}$ away from the end of the vacuum pipes. Signals were transmitted by Lemo and RG-58 cables and a BNC vacuum feedthrough. Results on proton energies (up to $1.6 \mathrm{MeV}$ ) and spectral distributions have been reported previously [35]. The maximum energies obtained from aluminium and mylar targets where of similar magnitude. Particle energies and spectral distributions are consistent with parametric tendencies of previous publications and with TNSA as the accelerating mechanism in the laser-plasma interactions.

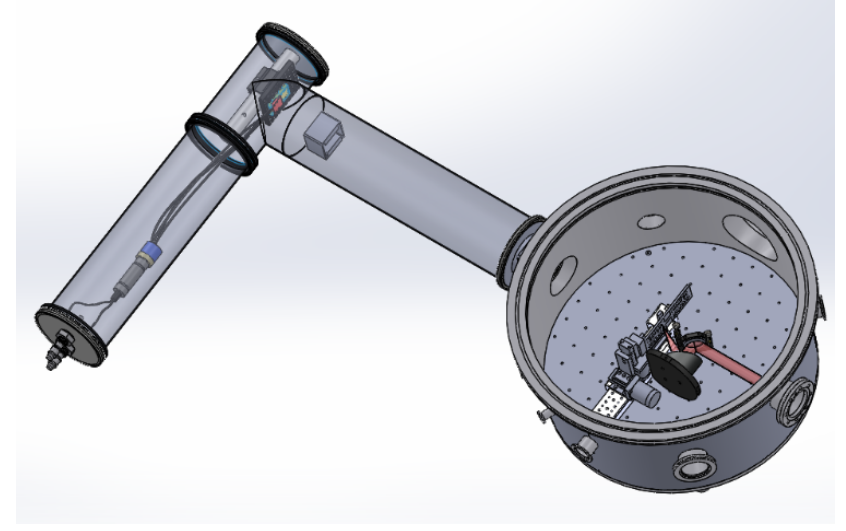

Figure 2. Sketch of the experimental setup (not to scale) comprising the main parts of the vacuum system.

Standard techniques for EMP detection comprise the use of Moebius loop antennas [17, 27,30], monopole antennas [37], and B-dot or D-dot probes [9, 10,23, $32,33]$. These allow for the measurement of absolute electric or magnetic field strengths inside or outside the vacuum vessel. As detailed in section 3, in our experiment the EMP signal was observed through its interference with the PMT anode pulses. On their way to the oscilloscope the rf waves are subject to frequency-dependent reflection, diffraction, delay, and absorption and by consequence, the measured signals can differ significantly from the original EMP. Nevertheless, our method is sufficiently precise for the relative comparison of noise amplitudes from different target materials as long as the same experimental and data analysis procedures are applied. In addition, the superposition with the TOF signal allows us to study the temporal structure of the rf component in relation to the time scales of particle detection, providing insight into the complete physical processes.

\section{Signal separation and pulse height analysis}

Typical oscilloscope signals from the TOF detector reveal the structure shown in Fig. 3(a)/(c) with a first, narrow peak caused by energetic photons and relativistic electrons from the laser-plasma interaction (referred to as "e $/ \gamma$ pulse"). This peak was used as oscilloscope trigger and has been chosen as zero point of our time scale throughout this analysis. Protons produce a second, much longer pulse starting about 110-150 ns after the first one. These signals have been used to obtain the maximum proton energies reported previously [35].
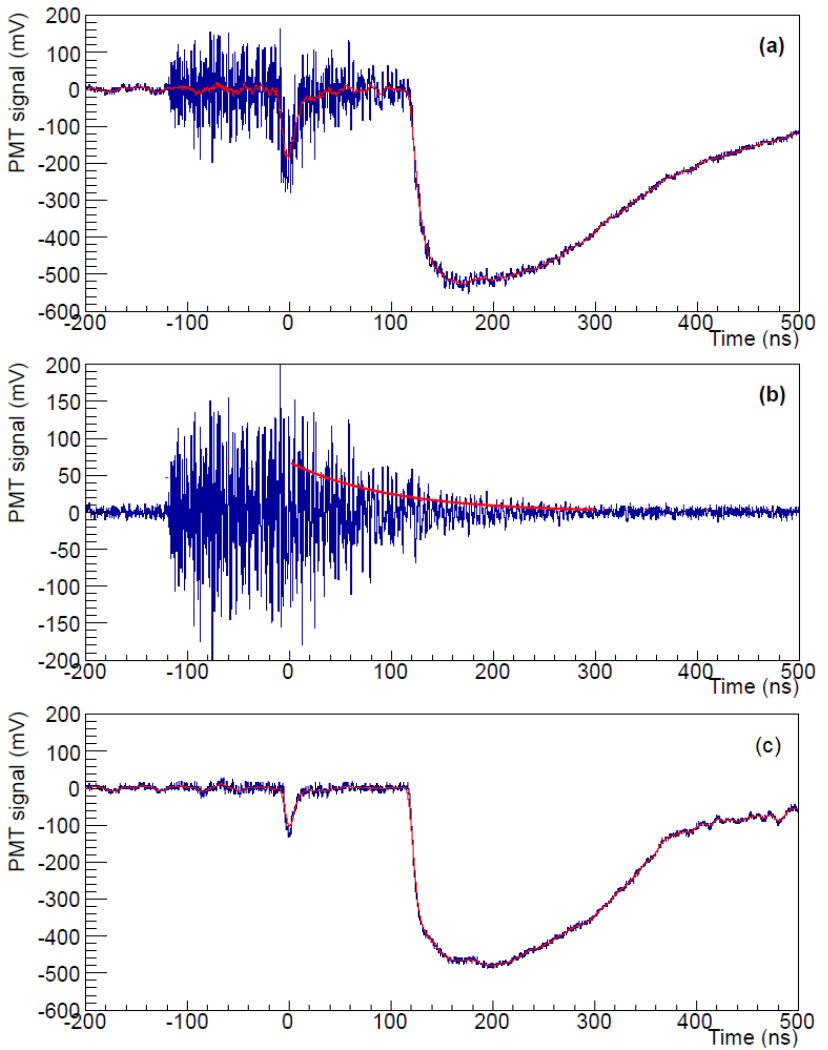

Figure 3. (a) TOF signal from a laser shot on a $2.5 \mu \mathrm{m}$ thick, elongate aluminium target foil. The red line represents the mean signal level. (b) EMP signal of (a) after subtraction of the mean signal. Here, the red line indicates the exponential decrease of the noise amplitude. (c) TOF signal from a laser shot on a $6 \mu \mathrm{m}$ thick, elongate mylar target foil.

The TOF signal is modulated by radiofrequency oscillations originating from a strong electromagnetic pulse. In Fig. 3(a), the onset of the rf component 
lies around $t_{\mathrm{rf}}=-120 \mathrm{~ns}$, long before the $e / \gamma$ pulse. We verified that this time interval increases with increasing length of the RG-58 cable connecting the PMT anode signal from the vacuum feedthrough to the oscilloscope. The rf signal is visible even with the PMT switched off. This implies that the rf oscillations are not emitted by the PMT or its anode circuitry and that not all of the EMP noise is transmitted through the coaxial cable. Instead, the oscilloscope picks up electromagnetic waves propagating through the air. Similar observations of EMP interference with detector signals through atmospheric transmission have been reported previously [6,27].

The Pockels cells of high-power laser systems are a potential source of $\mathrm{rf}$ noise [38]. In our setup the Pockels cells were placed inside Faraday cages. In order to determine a possible contribution to our signals several laser shots were performed under the same experimental conditions as in the series reported here, but the beam was absorbed before the compressor gratings and thus did not interact with the target foil. The remaining noise levels on the oscilloscope were insignificant as compared to those shown in Fig. 3.

The radiofrequency noise can be separated from the underlying $e / \gamma$ and proton pulses by subtraction of the mean pulse height (red line in Fig. 3(a)), resulting in an approximately constant level modulated by the rf component, Fig. 3(b). For a direct comparison between signals from different target materials the rf amplitudes have been analysed. Here, we refer to a measure for the height of the noise signal on the oscilloscope (in $\mathrm{mV}$ ), not the electric or magnetic field strengths. The EMP noise shows an exponential decrease after the $e / \gamma$ pulse $(t>0 \mathrm{~ns})$. A fit to

$A(t)=A_{0} \cdot e^{-t / t_{r}}+A_{e l}$

allows for extracting a maximum amplitude, $A_{0}$, and the relaxation time, $t_{r}$, for each recorded oscilloscope signal. The last term, $A_{e l}$, represents a constant electronic noise level independent of the EMP. In our experiments with multi-shot targets, EMP signal amplitudes have been obtained from aluminium foils between 1.8 and $7 \mu \mathrm{m}$ thickness, with 25-37 individual laser shots on each of them. Average values for $A_{0}$ are shown in Fig. 4(a). Under the same experimental conditions $A_{0}$ has been obtained for a $6 \mu \mathrm{m}$ mylar foil giving a much smaller mean value as expected from Fig. 3(c). The same procedure has been applied with single-shot targets for a wider range of thicknesses, Fig. 4(b). Here too, the EMP amplitudes from pure mylar are significantly smaller than those from aluminium. With aluminised mylar EMP levels similar to the ones of aluminium are observed. These data indicate that the target material and, more precisely, the presence of an aluminium layer is the most decisive factor for the noise amplitudes. The target thickness, to the contrary, does not correlate with any systematic trends. It is tentative to attribute the observed differences to the conductivity of a metallic foil versus the dielectric nature of the polymer. This will be further discussed in section 5. A comparison of the EMP amplitudes from aluminium of Figs. 4(a) and 4(b) reveals systematically larger values with multishot foils. These seem to be related to the target area which was the parameter with the biggest difference between the two series.
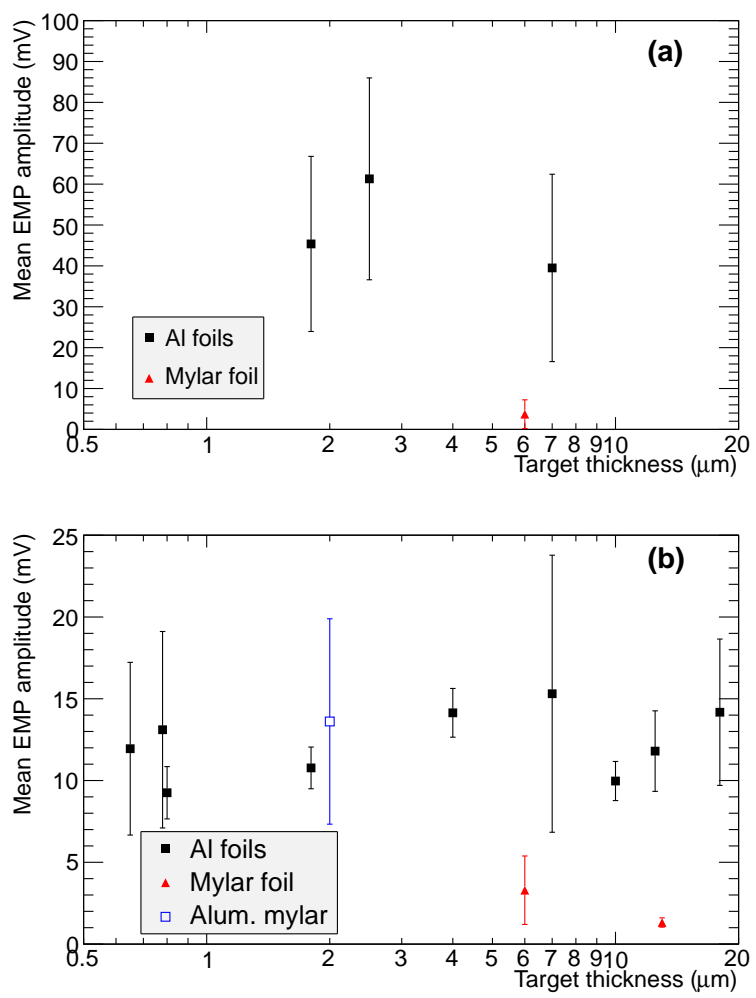

Figure 4. Fitted rf amplitudes, $A_{0}$, for targets of different thicknesses, averaged over several laser shots. (a) Multi-shot, (b) single-shot foils.

In our PMT anode signals, Fig. 3 , the $e / \gamma$ pulse at $t=0$ is produced by energetic photons and particles close to light speed (MeV-electrons). For aluminium targets the height of this pulse is correlated to the EMP amplitude (Fig. 5); the two, a priori independent, quantities increase in parallel (a linear fit is shown to guide the eye). This observation supports the importance of relativistic electrons as one possible source of rf emission [18], yet without providing a detailed explanation of the excitation mechanism (e.g., through their wakefield or upon impact on metallic structures). It is further observed that the peak amplitudes from mylar targets do not follow the trend indicated by the data points from aluminium foils. We interpret these findings in the way that the contribution of energetic electrons to the $e / \gamma$ peak 
is suppressed in the case of mylar foils. For the physical processes of the laser-plasma interaction this hints towards are stronger trapping of electrons in the rear sheath field for dielectric targets as compared to conductors [25]. The high mobility of charges in conductive materials allows bulk electrons to rapidly replace higher energetic electrons that escape from the sheath field, an effect which is suppressed for dielectrics.

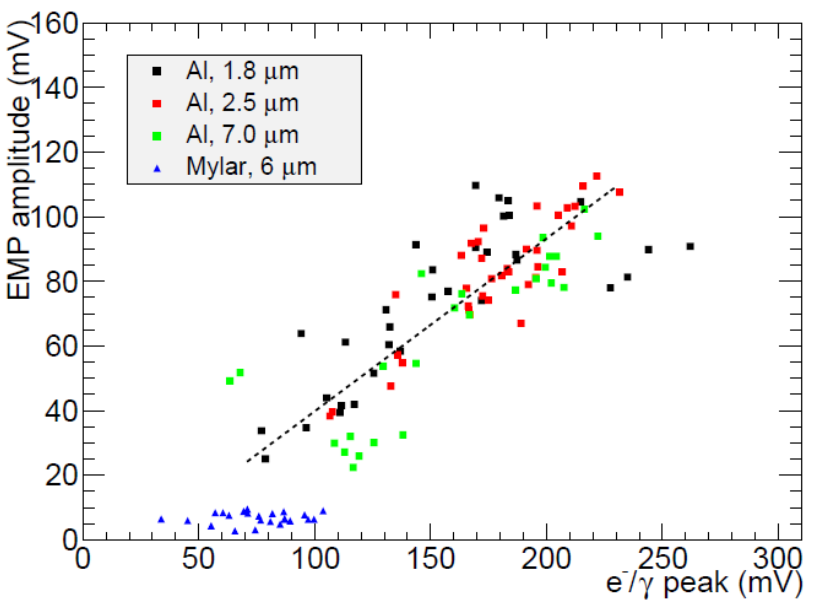

Figure 5. Scatter plot of EMP amplitudes vs. the height of the $e / \gamma$ pulse for multi-shot data. The dashed line is a linear fit to the $\mathrm{Al}$ data points.

\section{Frequency analysis and sources of $\mathrm{rf}$ radiation}

In laser-plasma interactions, the excitation of eigenmodes of the vacuum system has been suggested as a source of rf radiation in the $\mathrm{MHz}-\mathrm{GHz}$ regime $[17,18]$. This may occur when a cloud of ions from the evaporating target expands and eventually collides with the vacuum vessel or other, internal components of the experimental setup [33]. In addition, the bunch of relativistic electrons escaping the hot plasma and the strong electromagnetic field behind the target foil induces wakefields in the vacuum vessel and pipes which then emit their characteristic eigenfrequencies [39]. In order to analyse the contribution of such discrete modes we have performed a Fast Fourier Transform (FFT) of the subtracted noise signals. Several pronounced peaks can be observed from aluminium targets, Fig. 6(a). Peak regions selected for further analysis are highlighted with colours. With mylar foils, Fig. 6(b), some peaks are visible as well but their amplitudes tend to be an order of magnitude smaller as compared to aluminium. With mylar, a peak in the interval $0-0.15 \mathrm{GHz}$ appears large as compared to the others; it shows similar, absolute amplitudes with aluminium as well. It is not visible with the PMT switched off. We therefore conclude that it originates from electronic noise of the PMT anode and it will not be considered in the remainder of this analysis.

The target vessel and vacuum pipes resonate approximately like cylindrical cavities. Their eigenmodes correspond to standing wave patterns of microwaves and are commonly denoted transverse electric $\left(\mathrm{TE}_{\varphi r z}\right)$ and transverse magnetic $\left(\mathrm{TM}_{\varphi r z}\right)$, with indices $\varphi, r$, $z$ indicating the numbers of nodes in cylindrical coordinates [40]. The fundamental frequency of the target chamber with radius $a=0.3 \mathrm{~m}$ can be calculated analytically as

$f_{0}=\frac{2.405 c}{2 \pi a}$

with $c$, the speed of light in vacuum. This $\mathrm{TM}_{010}$ mode at $0.38 \mathrm{GHz}$ is shifted towards somewhat higher frequencies $(0.42-0.45 \mathrm{GHz})$ when details of the experimental setup are considered, such as the glass cover of the vessel. This has been done by a numerical simulation of the eigenfrequencies of the setup using the multiphysics software COMSOL. The simulated geometry comprises various vacuum pipes as well as internal components such as the target holder and the TOF detector with its support; details have been presented elsewhere [41]. Several eigenmodes of the target chamber follow at higher frequencies. Some of them, which may be identified with the highlighted peaks of Fig. 6(a), are summarized in Table 1. The lowest mode of the vacuum tube containing the timeof-flight detector is at $1.18 \mathrm{GHz}$. Peaks visible in some of the laser shots, with frequencies higher than $1.18 \mathrm{GHz}$, are not discussed here because in this interval the density of simulated eigenmodes is too high to relate the corresponding sources to the data. In addition, internal components acting as antennas contribute to the spectrum with frequencies below or above $f_{0}$. In the latter case they may be close to an eigenmode of the vessel and therefore complicate the interpretation of the experimental data due to the finite width of the observed peaks. In total, our simulation has revealed about 500 eigenmodes between 0 and $2.5 \mathrm{GHz}$.

We have analysed data records from two experimental series. The first one, from single-shot foils on aluminium (0.8-18 $\mu \mathrm{m}, 31$ shots), pure mylar (6-13 $\mu \mathrm{m}$, 15 shots $)$, and aluminised mylar (2 $\mu \mathrm{m}, 5$ shots). And the second one, from multi-shot foils with a total of 98 shots on aluminium targets (1.8-7 $\mu \mathrm{m}$ thickness) and 24 shots on a pure mylar foil $(6 \mu \mathrm{m})$. The results presented here refer to observations made consistently throughout these data sets or major subsets of these samples. In order to extract quantitative information from the frequency spectra we have calculated integral values, normalised to the width of the peaks, around the peak positions defined in Table 1. Results are plotted in 


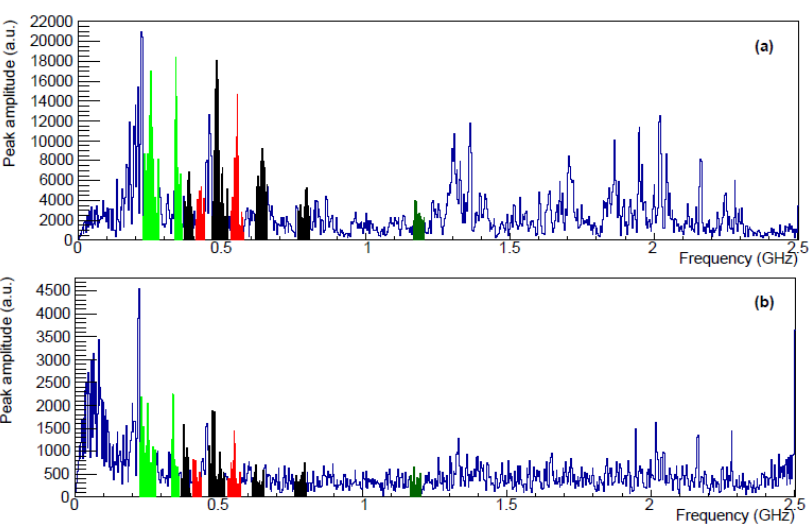

Figure 6. Fourier spectra of subtracted signals from multi-shot target foils of (a) $2.5 \mu \mathrm{m} \mathrm{Al}$, (b) $6 \mu \mathrm{m}$ mylar.

\begin{tabular}{|ccl|}
\hline$f_{\exp }(\mathrm{GHz})$ & $f_{\text {sim }}(\mathrm{GHz})$ & Relation to components \\
\hline 0.250 & 0.234 & TOF detector stand \\
0.250 & 0.238 & Target holder \\
0.345 & 0.312 & OAP with stand \\
0.380 & 0.382 & Chamber, TM \\
0.420 & 0.455 & Chamber, $\mathrm{TM}_{010}$ \\
0.490 & 0.503 & Chamber, $\mathrm{TM}_{110}$; int. \\
0.550 & 0.579 & Chamber, $\mathrm{TE}_{111}$ \\
0.635 & 0.609 & Chamber, $\mathrm{TM}_{011}$ \\
0.635 & 0.664 & Chamber, $\mathrm{TM}_{110}$ \\
0.635 & 0.673 & Chamber, $\mathrm{TE}_{111}$ \\
0.780 & 0.781 & Chamber, $\mathrm{TM}_{111}$ \\
0.780 & 0.793 & Chamber, $\mathrm{TE}_{011}$ \\
1.180 & 1.177 & Flight tube, $\mathrm{TE}_{111}$ \\
\hline
\end{tabular}

Table 1. Eigenfrequencies (central values, $f_{\text {exp }}$ ) selected for our analysis and their relation to specific components. The frequency interval covered by a single peak in FFT spectra may correspond to one or more calculated modes, $f_{\text {sim }}$.

Fig. 7. Again, only the relative variations between targets at identical frequencies are addressed; these are not affected by eventual, frequency-dependent calibration factors of the detection method. For aluminium, the integrals are much larger from multi-shot foils as compared to single-shot targets. This is presumably related to the target areas as all the other experimental parameters were approximately kept constant throughout both series. With multi-shot foils a big difference between aluminium and mylar targets can be seen for all the peaks. With single-shot foils, the peaks between 0.55 and $0.78 \mathrm{GHz}$, attributed to resonance frequencies of the target vessel, are not prominent. The other ones, again, show clear dissimilarities between aluminium and mylar. Interestingly, the values from aluminised mylar are generally much closer to the ones of aluminium than to pure mylar. This confirms the observations of Fig. 4. In addition, it shows that the breach between aluminium and mylar is not explained by a single frequency interval but rather by the entire spectrum.
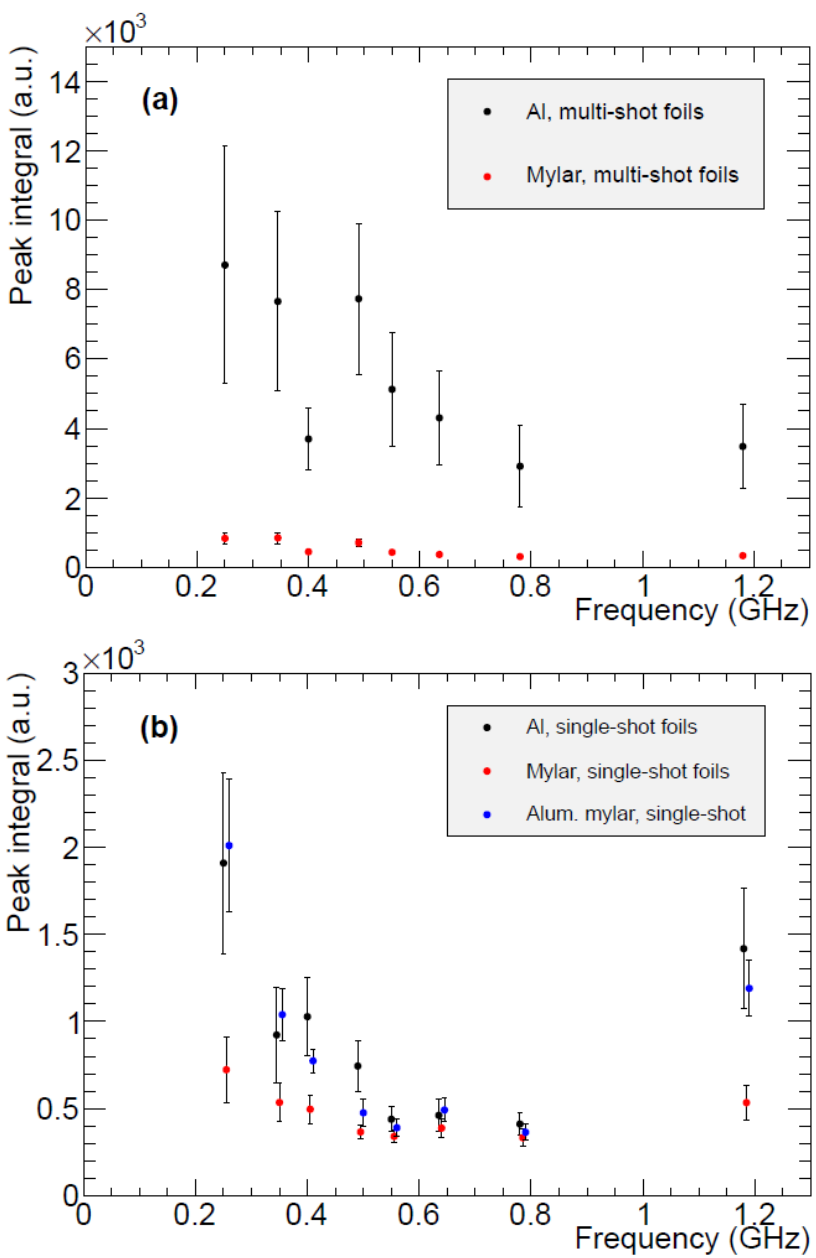

Figure 7. Peak integrals (mean values and standard deviations over available data sets). (a) Multi-shot, (b) single-shot targets.

\section{Time analysis at single frequencies}

With the aim of studying the temporal evolution of certain eigenmodes we have performed an inverse FFT of discrete frequency intervals (the complex FFTs implemented in the analysis software ROOT [42] preserve the phase information). As an example, a cut on the shaded region around $0.490 \mathrm{GHz}$ has been applied on the spectra of Fig. 6, eliminating all other frequencies. An inverse FFT then gives rise to the respective time distributions of Fig. 8 showing a maximum intensity close to $-30 \mathrm{~ns}$ approximately.

For each of the peaks of Table 1 we have found consistent maxima in the inverse FFTs throughout our data series on multi-shot targets made of aluminium (here, no distinction is made between foils of different thicknesses). The positions of these maxima are indicated as horizontal bars in Fig. 9; they represent 


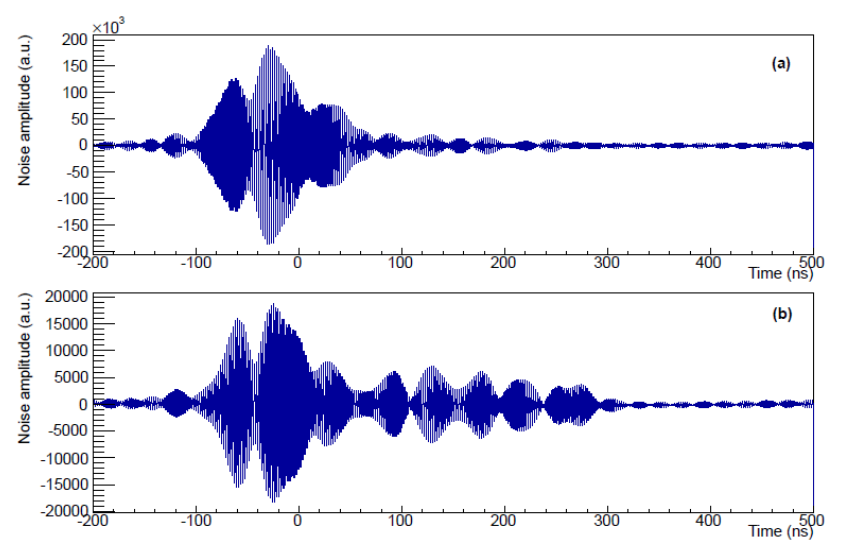

Figure 8. Temporal evolution of rf radiation at $0.49 \mathrm{GHz}$ from multi-shot target foils of (a) $2.5 \mu \mathrm{m} \mathrm{Al}$, (b) $6 \mu \mathrm{m}$ mylar.

the $\pm 1 \sigma$ intervals around the mean values of shots on the same target material. In some cases not only one maximum is present in the data, corresponding to a larger width in this representation. At $0.25 \mathrm{GHz}$, $0.345 \mathrm{GHz}$, and $0.49 \mathrm{GHz}$ the peaks in multi-shot data on mylar are sufficiently prominent to apply the same analysis procedure. Their time maxima coincide with those of aluminium targets within the experimental errors, indicating that the physical excitation processes are the same despite the large differences in amplitudes observed previously. In the single-shot data on aluminium foils, only three peaks show clear maxima in their temporal evolution. Two of them (at $0.38 \mathrm{GHz}$ and $1.18 \mathrm{GHz}$ ) coincide with the corresponding multishot ones, again underlining the overall consistency between different data sets. At $0.25 \mathrm{GHz}$ the data from single-shot foils seem to be at variance with the multi-shot series, however the time distribution in this case is not limited to a single, dominant maximum and comprises an early contribution as well.

In order to relate the frequency components of the recorded signals to their physical sources the time delays related to different transmission lines (TLs) from the source to the oscilloscope have to be taken into account. Their typical time scale is of the order of tens of nanoseconds, far beyond the laser-plasma interaction and the particle acceleration process $(<1 \mathrm{ps})$. This holds as well for the detection of particles. More precisely, a particle accelerated at time $t_{0}$ (laser incidence) and hitting the scintillator will produce scintillation photons propagating to the PMT which anode pulse is guided through coaxial cables. All the components of this TL1 contribute to the corresponding pulse time on the oscilloscope via

$t=t_{0}+t_{\mathrm{TOF}}+t_{\text {fiber }}+t_{\mathrm{PMT}}+t_{\text {int }}+t_{\text {cable }}$

with the time-of-flight, $t_{\mathrm{TOF}}$, the transmission time of scintillation photons through the optical fibers, $t_{\text {fiber }} \simeq$ $5 \mathrm{~ns}$, the $\mathrm{PMT}$ transient time, $t_{\mathrm{PMT}} \simeq 22 \mathrm{~ns}$, the delay caused by the anode cable inside the vacuum system, $t_{\text {int }}=5 \mathrm{~ns}$, and the exterior RG-58 cable of $15 \mathrm{~m}$ length, $t_{\text {cable }}=75 \mathrm{~ns}$. For light-speed particles $\left(t_{\mathrm{TOF}}=\right.$ $7.5 \mathrm{~ns}$ ) this sums up to about $115 \mathrm{~ns}$, accounting approximately for the observed delay between the onset of EMP noise and the $e / \gamma$ pulse.

The transmission of radiofrequency waves from their respective sources to the oscilloscope can follow two alternative paths. One of them (TL2) is directly through the atmosphere with a detection at

$t=t_{0}+t_{\mathrm{ex}}+t_{\mathrm{air}}$

and $t_{\text {air }} \simeq 10$ ns. $t_{\text {ex }}$ refers to the time of excitation as detailed below. Further, electromagnetic waves can be conducted through the vacuum chamber and pipes and the exterior RG-58 cable (TL3) with a corresponding signal at

$t=t_{0}+t_{\mathrm{ex}}+t_{\mathrm{vac}}+t_{\text {cable }}$.

In this case we estimate $t_{\mathrm{vac}} \simeq 10 \mathrm{~ns}$ for tubes of about $3 \mathrm{~m}$ length and the dominating delay between the physical excitation and the detection on the oscilloscope is given by the RG- 58 cable length.

These signal delays allow for estimating the temporal sequence of single noise frequencies as follows. Relativistic electrons may excite rf eigenmodes only during the first nanoseconds after the laser-plasma interaction, before or upon collision with some part of the setup. Neutralisation currents inside the target holder also appear immediately after the laser pulse and vanish quickly. On our time scale these two important sources of EMP noise can be considered instantaneous after laser incidence. The corresponding rf waves may reach the oscilloscope directly through the air (TL2) or through the vacuum system and the coaxial cable (TL3); the latter implies a significant delay. In Fig. 9 this gives rise to the two intervals shaded in green considering reasonable uncertainties for signal transmission. Opposed to that, an excitation by low-energy ions from the evaporating target bulk material which take several tens of nanoseconds to arrive at the vacuum chamber or internal components will produce later signals on the oscilloscope, indicated by the orange shaded intervals, again with the corresponding delays of TL2 and TL3. Here, for the flight times of the ions an interval of 40-60 ns has been assumed. For a distance of $0.3 \mathrm{~m}$ (the radius of the target vessel) this corresponds to a kinetic energy around $190 \mathrm{keV} / u$, a reasonable value for the plasma temperature. Protons are accelerated predominantly in target normal direction to enter the vacuum pipe and therefore are less likely to hit the walls of the target vessel. One can see that the time intervals of the peaks overlap with the shaded areas. Some of the frequencies attributed to eigenmodes of the vacuum vessel favour an excitation by low-energy 
ions (at $0.38 \mathrm{GHz}, 0.55 \mathrm{GHz}$, and $0.78 \mathrm{GHz}$ ) while others coincide rather with instantaneous emission (0.345 GHz, $1.18 \mathrm{GHz})$.

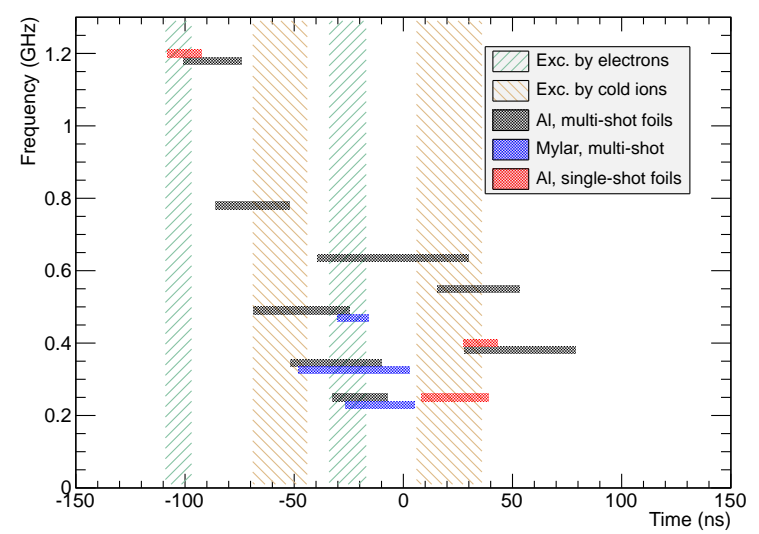

Figure 9. Maxima in time of inverse FFTs for single peaks.

In Fig. 6 a prominent peak is observed at $1.18 \mathrm{GHz}$. Its corresponding maximum in time (Fig. 9) is between $-100 \mathrm{~ns}$ and $-80 \mathrm{~ns}$, long before most of the other ones. As a matter of fact, in some shots we found additional peaks at $1.30-1.35 \mathrm{GHz}$, sometimes with larger amplitudes than the one at $1.18 \mathrm{GHz}$, with their maxima close to $-100 \mathrm{~ns}$ as well. This temporal position can only be understood by a transmission of rf waves through the atmosphere (TL2). The frequency of $1.18 \mathrm{GHz}$ coincides with the fundamental mode $\left(\mathrm{TE}_{111}\right)$ of the vacuum pipe guiding to the TOF detector. Our data do not allow for determining if this mode is excited by the passage of relativistic electrons or if this rf radiation rather corresponds to EMP directly from the target region. Neutralisation currents which have been shown to oscillate on a typical time scale of 1 ns $[9,21,22]$ are consistent with this component as well. Our observation that this frequency is not prominent with mylar targets may be in line with a suppression of these currents for isolated targets as predicted by Poyé et al. [23]. However, a more rigorous interpretation is not possible at this point as our setup did not comprise means for the direct measurement of the target currents. Note that any contribution to these early peaks from other possible sources of rf emission, e.g., the Pockels cells, would be of equal magnitude for all target materials, which is not supported by the data of Fig. 7 .

In the data from a multi-shot mylar foil, the peaks which show a clear distribution in time (at $0.25 \mathrm{GHz}$, $0.345 \mathrm{GHz}$, and $0.49 \mathrm{GHz}$ ) are consistent with instantaneous excitation. These peaks are an order of magnitude smaller than from $\mathrm{Al}$ targets, Fig. 7(a). The excitation of vacuum chamber resonances by lowenergy ions seems to be important only for aluminium targets; the corresponding peaks are not prominent with mylar foils.

\section{Conclusions}

We present data from two series of laser-ion acceleration experiments to analyse the radiofrequency noise which was emitted after the laser-plasma interaction and interfered with oscilloscope signals from a time-offlight detector. We have observed differences of a factor $\sim 5$ in the EMP noise amplitudes from aluminium target foils of distinct lateral dimensions. No systematic effects of the target thickness within each of the series have been seen. The most striking observation is that, under otherwise identical conditions, pure mylar foils within the range of thicknesses covered by those made of aluminium produced significantly lower EMP noise amplitudes (a factor 5-10).

A frequency analysis of the noise signals reveals peaks with coherent positions among different data sets. Some prominent peaks can be related to eigenmodes of the cylindrical target chamber, in agreement with previous work [17]. Others, which are clearly below the fundamental frequency of the vessel, are attributed to internal parts such as the target holder. The arrival times on our oscilloscope can differ by more than $100 \mathrm{~ns}$, far beyond the duration of the laser-plasma interaction processes. This is interpreted in terms of two distinct time scales for rf emission, one within the first nanoseconds after the laser pulse due to neutralisation currents or relativistic electrons, and another one due to ions from the evaporating aluminium target hitting the target chamber. They can roughly be related to the measured times taking into account the possible paths of signal transmission to the oscilloscope.

These dissimilarities between EMP amplitudes from conductive and dielectric targets may be attributed to the trapping of recirculating electrons, variations in the numbers of ions from the evaporating bulk material, and the suppression of target neutralisation currents as predicted by models of target charging in interactions with short laser pulses [25].

In our experiments we obtained similar proton spectra from aluminium or mylar targets. Our measurements suggest that, in the context of EMP suppression in laser acceleration, the use of dielectric target materials with limited areas can be advantageous.

\section{Acknowledgments}

This project has been funded by Ministerio de Economía y Competitividad, ref. RTC-2015-32781, with further support by Ministerio de Ciencia, 
Innovación y Universidades, ref. RTI2018-101578-B$\mathrm{C} 22$.

\section{References}

[1] Daido H, Nishiuchi M and Pirozhkov A S 2012 Reports on Progress in Physics $\mathbf{7 5} 056401$

[2] Macchi A, Borghesi M and Passoni M 2013 Rev. Mod. Phys. 85 751-793

[3] Wilks S C, Langdon A B, Cowan T E, Roth M, Singh M, Hatchett S, Key M H, Pennington D, MacKinnon A and Snavely R A 2001 Physics of Plasmas 8 542-549

[4] Roth M and Schollmeier M 2016 CERN Yellow Reports 1 231

[5] Ledingham K, Bolton P, Shikazono N and Ma C M 2014 Applied Sciences 4 402-443

[6] Remo J L, Adams R G and Jones M C 2007 Appl. Opt. 46 $6166-6175$

[7] A Aspiotis J, Barbieri N, Bernath R, G Brown C, Richardson M and Y Cooper B 2006 Proc. SPIE 6219 621908

[8] Jinwen Y, Tingshuai L, Tao Y, Chuanke W, Ming Y, Weiming Y, Shenye L, Shaoen J and Yongkun D 2016 Plasma Science and Technology 181044

[9] Raczka P, Dubois J L, Hulin S, Tikhonchuk V, Rosiński M, Zaraś-Szydłowska A and Badziak J 2017 Laser and Particle Beams 35 677-686

[10] Robinson T S, Consoli F, Giltrap S, Eardley S J, Hicks G S, Ditter E J, Ettlinger O, Stuart N H, Notley M, Angelis R D, Najmudin Z and Smith R A 2017 Scientific Reports 7983

[11] Consoli F, Angelis R D, Robinson T, Giltrap S, Hicks G, Ditter E, Ettlinger O, Najmudin Z, Notley M and Smith R 2019 Scientific Reports 98551

[12] Lazarus J, Nilson P M, Smith R A, Moore A S, Clark E L, Eagleton R T, Edwards R D, Gumbrell E T and Clarke R J 2005 Electromagnetic pulse suppression in laser plasma interaction experiments on the vulcan petawatt laser Tech. Rep. Central Laser Facility Annual Report 2004/2005 STFC Rutherford Appleton Laboratory

[13] Stoeckl C, Glebov V Y, Jaanimagi P A, Knauer J P, Meyerhofer D D, Sangster T C, Storm M, Sublett S, Theobald W, Key M H, MacKinnon A J, Patel P, Neely D and Norreys P A 2006 Review of Scientific Instruments 77 10F506

[14] Bourgade J L, Marmoret R, Darbon S, Rosch R, Troussel P, Villette B, Glebov V, Shmayda W T, Gomme J C, Tonqueze Y L, Aubard F, Baggio J, Bazzoli S, Bonneau F, Boutin J Y, Caillaud T, Chollet C, Combis P, Disdier L, Gazave J, Girard S, Gontier D, Jaanimagi P, Jacquet H P, Jadaud J P, Landoas O, Legendre J, Leray J L, Maroni R, Meyerhofer D D, Miquel J L, Marshall F J, Masclet-Gobin I, Pien G, Raimbourg J, Reverdin C, Richard A, de Cervens D R, Sangster C T, Seaux J P, Soullie G, Stoeckl C, Thfoin I, Videau L and Zuber C 2008 Review of Scientific Instruments 79 10F301

[15] Eder D C, Throop A, Jr C G B, Kimbrough J, Stowell M L, White D A, Song P, Back N, MacPhee A, Chen H, DeHope W, Ping Y, Maddox B, Lister J, Pratt G, Ma T, Tsui Y, Perkins M, O'Brien D and Patel P 2009 Mitigation of electromagnetic pulse (EMP) effects from short-pulse lasers and fusion neutrons Tech. Rep. LLNLTR-411183 Lawrence Livermore National Laboratory

[16] Kar S, Ahmed H, Prasad R, Cerchez M, Brauckmann S, Aurand B, Cantono G, Hadjisolomou P, Lewis C, Macchi A, Nersisyan G, Robinson A, Schroer A, Swantusch M, Zepf M, Willi O and Borghesi M 2016 Nature Communications 710792
[17] Mead M J, Neely D, Gauoin J, Heathcote R and Patel P 2004 Review of Scientific Instruments 75 4225-4227

[18] Bateman J E and Mead M J 2012 Electromagnetic pulse generation in petawatt laser shots Tech. Rep. RAL-TR2012-005 STFC Rutherford Appleton Laboratory

[19] Felber F S 2005 Applied Physics Letters 86231501

[20] Chen Z Y, Li J F, Li J and Peng Q X 2011 Physica Scripta 83055503

[21] Dubois J L, Lubrano-Lavaderci F, Raffestin D, Ribolzi J, Gazave J, Fontaine A C L, d'Humières E, Hulin S, Nicolaï P, Poyé A and Tikhonchuk V T 2014 Phys. Rev. E 89 013102

[22] Cikhardt J, Krása J, De Marco M, Pfeifer M, Velyhan A, Krouský E, Cikhardtová B, Klír D, Řezáč K, Ullschmied J, Skála J, Kubeš P and Kravárik J 2014 Review of Scientific Instruments 85103507

[23] Poyé A, Hulin S, Bailly-Grandvaux M, Dubois J L, Ribolzi J, Raffestin D, Bardon M, Lubrano-Lavaderci F, D'Humières E, Santos J J, Nicolaï P and Tikhonchuk V 2015 Phys. Rev. E 91043106

[24] Sprangle P, Peñano J R, Hafizi B and Kapetanakos C A 2004 Phys. Rev. E 69066415

[25] Poyé A, Dubois J L, Lubrano-Lavaderci F, D'Humières E, Bardon M, Hulin S, Bailly-Grandvaux M, Ribolzi J, Raffestin D, Santos J J, Nicolaï P and Tikhonchuk V 2015 Phys. Rev. E 92043107

[26] Poyé A, Hulin S, Ribolzi J, Bailly-Grandvaux M, LubranoLavaderci F, Bardon M, Raffestin D, Santos J J and Tikhonchuk V 2018 Phys. Rev. E 98033201

[27] De Marco M, Pfeifer M, Krousky E, Krasa J, Cikhardt J, Klir D and Nassisi V 2014 Journal of Physics: Conference Series $\mathbf{5 0 8} 012007$

[28] Miragliotta J A, Brawley B, Sailor C, Spicer J B and Spicer J W M 2011 Proc. SPIE $803780370 \mathrm{~N}-1$

[29] Varma S, Spicer J, Brawley B and Miragliotta J A 2014 Optical Engineering $\mathbf{5 3} 051515$

[30] Krása J, De Marco M, Cikhardt J, Pfeifer M, Velyhan A, Klír D, Řezáč K, Limpouch J, Krouský E, Dostál J, Ullschmied J and Dudžák R 2017 Plasma Physics and Controlled Fusion 59065007

[31] De Marco M, Krása J, Cikhardt J, Velyhan A, Pfeifer M, Dudžák R, Dostál J, Krouský E, Limpouch J, Pisarczyk T, Kalinowska Z, Chodukowski T, Ullschmied J, Giuffrida L, Chatain D, Perin J P and Margarone D 2017 Physics of Plasmas 24083103

[32] Kugland N L, Aurand B, Brown C G, Constantin C G, Everson E T, Glenzer S H, Schaeffer D B, Tauschwitz A and Niemann C 2012 Applied Physics Letters 101024102

[33] Bradford P, Woolsey N C, Scott G G, Liao G, Liu H, Zhang Y, Zhu B, Armstrong C, Astbury S and Brenner C 2018 High Power Laser Science and Engineering 6 e21

[34] Lera R, Bellido P, Sánchez I, Mur P, Seimetz M, Benlloch J M, Roso L and Ruiz-de-la Cruz A 2019 Applied Physics $B \mathbf{1 2 5}$

[35] Bellido P, Lera R, Seimetz M, de la Cruz A R, TorresPeirò S, Galán M, Mur P, Sánchez I, Zaffino R, Vidal L, Soriano A, Sánchez S, Sánchez F, Rodríguez-Álvarez M, Rigla J, Moliner L, Iborra A, Hernández L, Grau-Ruiz D, González A, García-Garrigos J, Díaz-Caballero E, Conde P, Aguilar A, Roso L and Benlloch J 2017 Journal of Instrumentation 12 T05001

[36] Seimetz M, Bellido P, Soriano A, López J G, JiménezRamos M C, Fernández B, Conde P, Crespo E, González A J, Hernández L, Iborra A, Moliner L, Rigla J P, Rodríguez-Álvarez M J, Sánchez F, Sánchez S, Vidal L F and Benlloch J M 2015 IEEE Transactions on Nuclear Science 62 3216-3224

[37] Consoli F, Angelis R D, De Marco M, Krasa J, Cikhardt J, Pfeifer M, Margarone D, Klir D and Dudzak R 2018 Plasma Physics and Controlled Fusion 60105006 
[38] Price C J, Donnelly T D, Giltrap S, Stuart N H, Parker S, Patankar S, Lowe H F, Drew D, Gumbrell E T and Smith R A 2015 Review of Scientific Instruments 86033502

[39] Weiland T and Zotter B 1981 Particle Accelerators 11 143151

[40] Ramo S, Whinnery J and van Duzer T 1965 Fields and Waves in Communication Electronics third edition 1993 ed (John Wiley and Sons)

[41] Seimetz M 2018 Proceedings of the 2018 COMSOL Conference Lausanne URL https://www.comsol.es/paper/rf-emission-spectra-in-laser-plasma-acceleration-of-protons-66301

[42] Brun R and Rademakers F 1997 Nuclear Instruments and Methods in Physics Research Section A: Accelerators, Spectrometers, Detectors and Associated Equipment 389 $81-86$ 